\title{
Learning and transposition of an extended sameness-difference concept by slow and fast learning capuchin monkeys
}

\author{
JOHN L. SCANLON and JAMES E. KING \\ University of Arizona, Tucson, Arizona 85721
}

\begin{abstract}
Capuchin monkeys were trained on a series of learning-set problems which required discrimination between stimulus arrays having $\mathrm{ABA}$ and $\mathrm{BBA}$ configurations. Transposition was later tested by presenting training configurations with $\mathrm{CCC}$ or $\mathrm{ABC}$ configurations. Considerable negative transposition and a small amount of positive transposition occurred, a result suggesting that the original learning set was based at least partly on perceived differences in the homogeneity of the ABA and BBA configurations. Fast-learning subjects manifested more persistent random responding and less position responding than did slow-learning subjects. This difference suggests that random responding during the initial stages of learning may be used as an index of attention.
\end{abstract}

During the past 25 years, a highly diversified variety of learning sets have been demonstrated in learning research on nonhuman primates (French, 1965; Miles, 1965). For some varieties of learning sets, such as discrimination learning-set, the positive and negative stimulus within a given problem are differentiated by a constant difference in object quality. However, for other types of learning sets, such as oddity and sameness-difference, the positive and negative stimuli within a problem are not defined by any constant stimulus quality of the positive and negative stimulus but by the relationship either between the positive and negative stimuli, or between the stimuli within the positive or negative complexes.

King and Fobes (1975) demonstrated that capuchin monkeys could learn a sameness-difference learning set which required discrimination between simultaneously presented same (AA) and different (AB) object pairs with a new object combination appearing on each trial. Previously, King (1973) found that after learning a sameness-difference learning set, with the sameness pair (AA) positive, chimpanzees and organgutans preferred an $\mathrm{AAB}$ stimulus configuration over an ABA configuration. Conversely, after sameness-difference learning with the different (AB) pair positive, the apes preferred the ABA over the $\mathrm{AAB}$ configuration. Thus, although $\mathrm{AAB}$ and ABA configurations both contained two identical and one dissimilar element, the $\mathrm{AAB}$ configuration was apparently perceived by the apes as more homogeneous than the ABA configuration.

The above results suggest the question of whether monkeys can learn a modified sameness-difference learning set based upon discriminations between two

This research was partially supported by Training Grant MH-11286 from the United States Public Health Service. Requests for reprints should be sent to Dr. James E. King, Department of Psychology, University of Arizona, Tucson, Arizona 85721. configurations, each containing two identical and one.dissimilar object. The only difference would be that in the sameness configuration (BBA) the two identical objects would be spatially contiguous, while in the difference configuration (ABA) they would be separated by the dissimilar object.

Experiment 1 was designed to measure acquisition of this type of sameness-difference learning set in capuchin monkeys and, in particular, to permit application of a modified hypothesis analysis (Levine, 1965; King \& Fobes, 1975) to the data. This technique allows measurement of both random and nonrandom response patterns during acquisition. We were particularly interested in observing differences in systematic responding between slow and fast learning monkeys, since King and Fobes had previously observed such differences. Experiment 2 was addressed to the question of whether the samenessdifference learning in Experiment 1 would lead to transposition along a homogeneity-heterogeneity dimension. This transposition would be based solely on the stimulus arrangements within the positive and negative configurations, not on the number of identical or dissimilar elements within each configuration, and thus would be consistent with King's (1973) finding that $A A B$ configurations were perceived as more homogeneous than ABA configurations.

\section{METHOD}

\begin{abstract}
Subjects
The subjects were five male and two female adult capuchin monkeys (Cebus apella) which had previously received a small amount of object discrimination experience. None had had any prior experience on tasks involving any type of samenessdifference discrimination or transposition.
\end{abstract}

Apparatus

All testing was conducted in a manually operated Wisconsin 
General Test Apparatus (WGTA). The stimulus objects used during pretraining were four small junk objects attached to flat $5 \times 5 \mathrm{~cm}$ wood bases. The stimulus objects used during testing in both Experiment 1 and Experiment 2 were 60 wood blocks $(3.8 \times 5.1 \times 3.8 \mathrm{~cm})$ divided into 20 sets of three identical blocks. Ten of the block sets were each painted a distinctive homogeneous color with no other added identifying characteristics. Each of the other 10-block sets was also painted a distinctive homogeneous color and had small relatively flat junk objects affixed to the faces of the objects.

The stimulus objects were placed in small Plexiglas bins which were positioned over the two foodwells on the stimulus presentation tray. During testing, subjects could manually displace either bin, thus exposing the foodwell beneath the bin.

\section{Procedure}

Testing. At the start of each trial, the forward opaque screen of the WGTA was lifted and the stimulus presentation tray slowly moved forward toward the subject. The subject then displaced one of the bins and if the choice was correct, retrieved a half raisin from the foodwell. Correction was not allowed. After the subject's response, the experimenter lowered the opaque screen and pulled back the stimulus presentation tray. The position of the positive stimulus was varied according to a semirandom schedule, with the restriction that all eight possible 3-trial sequences appeared exactly three times on successive 72-trial blocks.

Pretraining. All animals were initially trained on two object discriminations to a criterion of 10 successive correct responses. Sixty trials were presented each day.

Experiment 1. Subjects were presented a series of discriminations in which a $\mathrm{BAB}$ configuration was paired with an $\mathrm{AAB}$ configuration; the former configuration was always rewarded, and the latter was always not rewarded. Thus, the only differentiating characteristic of positive or rewarded configurations was that the two identical objects were spatially separated by the dissimilar object, whereas in the negative configurations the two identical objects were spatially contiguous. Trials were presented in three-trial problems, with only two sets of objects used on each problem. An example of stimulus presentation on a typical problem is as follows: $\mathrm{AAB}$ vs. $\mathrm{BAB}$ on Trial 1, AAB vs. $A B A$ on Trial 2, and ABA vs. BBA on Trial 3. Half of the problems were defined by stimulus objects differing in color only, while the other half were defined by stimulus objects with affixed junk objects. The two types of problems were presented alternately. Twelve problems, or 36 trials, were presented each test day for a total of 60 days.

Experiment 2. In this experiment, transposition test trials were interspersed with original training trials according to the method of equivalent stimuli. Specifically, 36 differentially reinforced trials were presented each day in a manner identical to that used in Experiment 1. In addition, after 9 of the 12 problems each day, a single transposition test trial was presented during which both foodwells were baited. Two basic types of test trials were presented: positive transposition trials, which entailed presentation of the old positive configuration (ABA) with a more heterogeneous one $(\mathrm{ABC})$, and negative transposition trials, which entailed presentation of the old negative configuration (BBA) with a completely homogeneous one (CCC). Each day, one type of test trial was presented five times and the other four times; the order was then reversed on the succeeding day. Testing continued for 20 days.

\section{RESULTS}

\section{Experiment 1}

Observation of the overall performance of the seven subjects showed a distinct separation between four male subjects who demonstrated strong learning and the remaining three subjects (one male and two female) who demonstrated little evidence of learning. Two-tailed $z$ tests indicated above-chance performance by each of the four learners $(p<.01)$ during the last 10 days, while none of the nonlearners achieved above-chance performance. Figure 1 shows the percentage of correct responses for both groups as a function of 10-day blocks.

Analysis of variance revealed significant overall increase in correct responding over trial blocks $[F(5,25)=18.82, p<.005]$ and significantly more correct responses in problems defined by homogeneously painted objects than in problems defined by stimulus objects with junk objects attached $[F(1,5)=$ $17.26, \mathrm{p}<.01]$. The Groups by Blocks interaction was significant $[F(5,25)=7.42, p<.005]$, reflecting the increased performance of the learners over trials and the negligible improvement of the nonlearners. The Groups by Object Type interaction was also significant $[F(1,5)=20.49, p<.01]$, since the learners showed better performance with the homogeneous objects $(63.2 \%$ correct) than with the junk faced objects $(57.6 \%$ correct) while the nonlearners showed virtually chance level performance with both classes of objects.

The data were analyzed by a hypothesis analysis technique similar to that originally developed by Levine (1965), although a different set of hypotheses

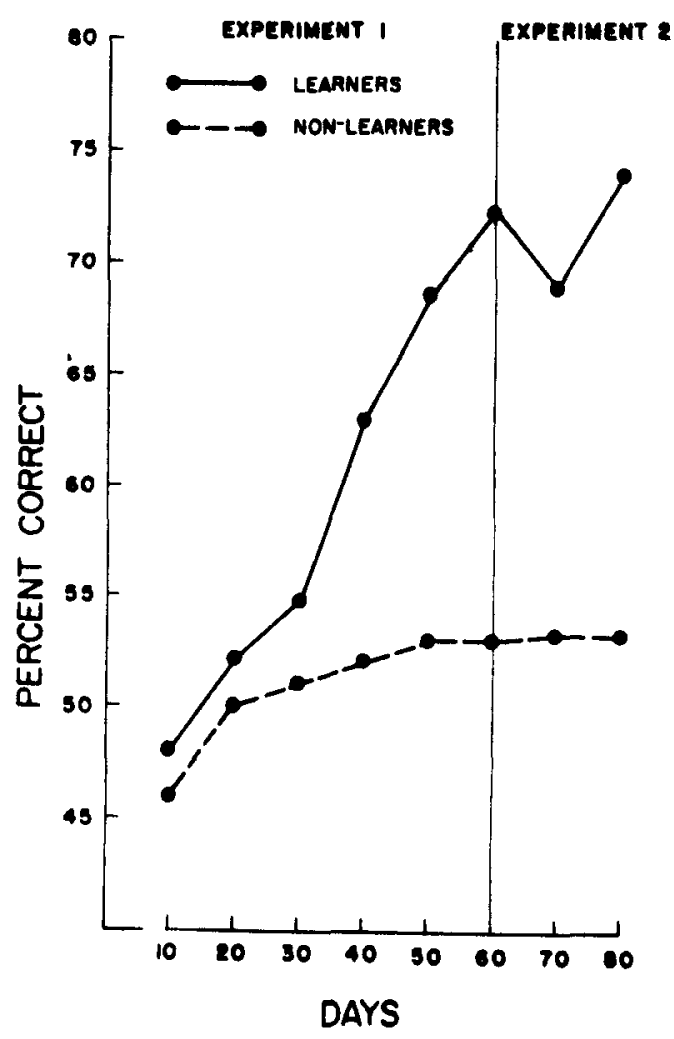

Figure 1. Percentage of correct responses made by learning and nonlearning subjects during Experiments 1 and 2. 
Table 1

Hypotheses in Experiment 1

\begin{tabular}{|c|c|c|}
\hline Hypotheses & Definitions & Manifestations \\
\hline Position Preference (P) & Sequence of Responses to One Side & I I l \\
\hline Doublet (D) & Two Consecutive Responses to One Side & I I O, 100 \\
\hline Correct (C) & Sequence of Correct Responses & +++ \\
\hline Two-Thirds Correct (T) & Two of Three Correct Responses &,,++-+-+-++ \\
\hline Incorrect (I) & Sequence of Incorrect Responses & --- \\
\hline $\begin{array}{c}\text { Win-Stay, Lose-Shift (W) } \\
\text { (Position) }\end{array}$ & Responding to Position Rewarded on Previous Trial & $\begin{array}{l}\mathrm{I}+\mathrm{I}+\mathrm{I}, \mathrm{I}+\mathrm{I}-\mathrm{O} \\
\mathrm{I}-\mathrm{O}+\mathrm{O}, \mathrm{I}-\mathrm{O}-\mathrm{I}\end{array}$ \\
\hline $\begin{array}{l}\text { Lose-Stay, Win-Shift (L) } \\
\text { (Position) }\end{array}$ & Responding to Position Not Rewarded on Previous Trial & $\begin{array}{l}\mathrm{I}-\mathrm{I}-\mathrm{I}, \mathrm{I}-\mathrm{I}+\mathrm{O} \\
\mathrm{I}+\mathrm{O}-\mathrm{O}, \mathrm{I}+\mathrm{O}+\mathrm{I}\end{array}$ \\
\hline Random (R) & Response Not Correlated with Stimulus Change & All Manifestations \\
\hline
\end{tabular}

+ indicates a correct response.

- indicates an incorrect response.

I indicates first position selected in the sequence.

$O$ indicates the other position.

was measured. Table 1 lists the hypotheses, their definitions, and their possible manifestations in three-trial sequences.

Of the eight hypotheses measured, only correct responding, $2 / 3$ correct responding, position preference, and random responding attained more than negligible strength. Changes in the strengths of these hypotheses over trials for the learners and nonlearners are depicted in Figure 2.

The learners achieved a significant increase in the correct $[F(5,15)=14.08, p<.005]$ and $2 / 3$ correct $[F(5,15)=4.40, p<.025]$ hypotheses, while the nonlearners did not, a result consistent with the basic learning curves of these two groups. Analysis of position preference showed that until the last trial block, the nonlearners retained a strong propensity for this hypothesis, the change over trials not being significant. The learners, however, manifested a fairly consistent decrease in position responding $[F(5,15)=2.92, p<.05)$. The Groups by Trials interaction was also significant $[F(5,25)=6.49$, $\mathrm{p}<.005$ ]. Random responding by the learners and nonlearners showed two different patterns of change across trials, as reflected in the significant Groups by Trials interaction $[F(5,25)=6.86, p<.005]$.
The learners manifested a fairly high level of random responding initially, with only a moderate decrease as learning progressed. The nonlearners, however, manifested a decline in random responding over the first three blocks, followed by a consistent increase.

\section{Experiment 2}

The nonlearners responded at virtually chance level on both types of transposition test trials. This result would be expected since these animals achieved only $54.9 \%$ correct responses on the nontest trials during Experiment 2.

The learners showed unmistakable evidence of negative transposition when the previously negative configuration (BBA) was paired with a totally homogenous configuration (CCC). On these trials, $72.5 \%$ of the responses were to the BBA configuration. This value was significantly greater than $50 \%[\mathrm{t}(3)=8.46, \mathrm{p}<.01]$. On positive transposition test trials, in which the previously positive configuration (ABA) was paired with a completely heterogeneous stimulus $(\mathrm{ABC}), 51.4 \%$ of the responses were to ABA. This percentage was not significantly different from $50 \%$, but it did differ
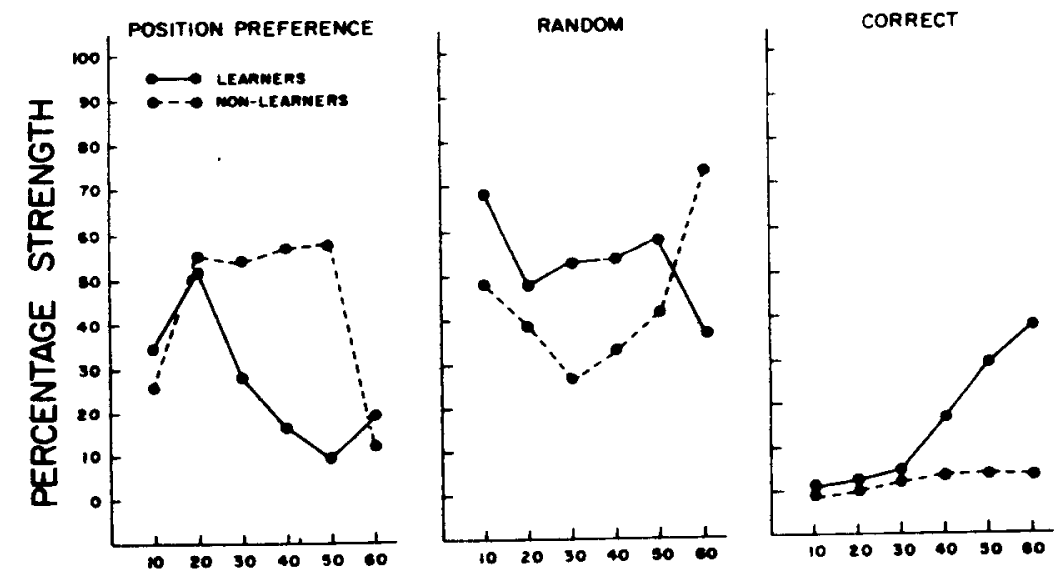

Figure 2. Strengths of position preference, random, and correct responding for learning and nonlearning subjects during Experiment 1. 
significantly $[\mathrm{t}(3)=10.8, \mathrm{p}<.01]$ from the $71.8 \%$ of the responses to the ABA configuration during the nontest trials in Experiment 2.

\section{DISCUSSION}

Experiment 1 demonstrated that the learner group of capuchin monkeys was able to acquire a learning set based on discrimination between $\mathrm{ABA}$ and $\mathrm{BBA}$ configurations with a wide variety of stimulus objects. Since both configurations contained two identical and one dissimilar element, the monkeys were obviously discriminating the arrangement of objects within the configurations and formed a learning set based not on oddity qualities alone, but on the position of an odd or different object within the three-object sets.

Performance was higher when the stimulus blocks were each painted a single color with no attached object than when objects were attached. Thus, discrimination of the position of the different objects within the arrays was impeded by the addition of relatively flat junk objects, and the result is therefore not only a lack of cue additivity but a subtractive effect. This result is an exception to the widely cited generalization that discriminations are learned at least as fast or faster with multidimensionall redundant stimuli than with unidimensional stimuli (see Warren \& McGonigle, 1969). For example, Meyer and Harlow (1949) observed that rhesus monkeys learned oddity problems defined by color differences at about the same rate as problems incorporating color, form, and size differences.

During Experiment 1, each monkey's performance fell distinctly into the learner or the nonlearner category. The marked separation of learning and nonlearning subjects was also observed by King and Fobes (1975), who tested a different group of capuchin monkeys on sameness-difference learningset problems. Furthermore, the difference in the pattern of hypothesis manifestations between learning and nonlearning monkeys was the same in both experiments. Learning subjects showed little evidence of position responding, but evidenced large and persistent random responding. Nonlearning subjects were highly susceptible to position preferences and showed relatively little random responding. Interestingly, the nonlearning subjects showed a sharp increase in random responding during the last trial block in Experiment 1, coincident with a decrease in position responding and a slight increase in correct responding. It is tempting to conclude that these changes in random and position responding by the nonlearners on the last trial block would have been precursors of a substantial increase in correct responding if the training trials had been further extended.

Thus, learning-set acquisition is obviously not always accurately described as a monotonic decrease in all error-producing hypotheses accompanied by a monotonic increase in correct responding, as was tacitly stated in past discussions of the subject. For example, Harlow (1959, p. 513) stated: "As these orderly but inappropriate responses are reduced, the percentage of errors diminishes progressively, and learning is said to take place."

The present study, as well as those of King and Fobes (1975) and Smith, King, and Newberry (1976), showed a correspondence between random and correct responding during learning-set acquisition and suggest a more complex evolution of hypothesis strengths during learning-set acquisition than that envisioned by Harlow. Three distinct stages are apparently involved: First, elimination of systematic error-producing hypotheses, mainly position preference; second, a relatively high level of random responding; and third, the acquisition of the correct hypothesis. In the present study, the first stage was rapidly mastered by the fast-learning monkeys, who then efficiently progressed to the third stage. The slow-learning monkeys were just entering the second phase at the end of Experiment 1.

The transposition tests in Experiment 2 revealed more negative than positive transposition, a frequently reported difference in past research on transposition (see Reese, 1968, for a review). This difference is also consistent with Spence's (1942) theoretical account of transposition in terms of overlapping approach and avoidance gradients established during training. This version of Spence's theory assumes that the approach gradient around the positive training stimulus has greater magnitude than the avoidance gradient around the negative training stimulus. This would indicate a greater absolute preference for the positive stimulus than absolute avoidance of the negative stimulus, which would result in the relatively small amount of positive transposition observed in the present study.

The results of the transposition test indicate that the monkeys during training may have learned to discriminate $\mathrm{ABA}$ and BBA arrays on the basis of a homogeneity-heterogeneity concept, since a perference for the more homogeneous array would result in both negative and positive transposition.

The strong negative and weak positive transposition observed in Experiment 2 would not have been expected if the monkeys were simply learning to approach the object array with the odd object on the end. Were that the case, the monkeys would have strongly preferred BBA over CCC on the negative transposition trials and would have either had no preference between $A B C$ and $A B A$, or had a slight preference for $A B C$ on positive transposition trials. Likewise, if the monkeys had learned only to approach the array with the most contiguous identical objects, a marked preference of CCC over 
BBA would have been observed, but little preference between $A B C$ and $A B A$ would have been expressed.

If the monkeys were consistently avoiding the new configurations (CCC or ABC) during the transposition test trials, negative but not positive transposition would occur. However, such avoidance of new configurations because of their unfamiliarity is probably not an explanation for the results of the transposition testing, since the $\mathrm{CCC}$ and $\mathrm{ABC}$ arrays always consisted of objects taken from the common object collection used for training, and therefore the subjects had had equal experience with the particular objects in both arrays presented on the transposition test trials. Only the object ordering within the $C C C$ and $A B C$ arrays was relatively unfamiliar to the monkeys, and this seems unlikely to evoke significant avoidance. Furthermore, the small amount of positive transposition that did occur is inconsistent with the unfamiliarity explanation.

Several years ago, Harlow (1959) noted that all transposition research up to that time entailed a transposition test given after single problem learning. Since there is considerable evidence that concept formation is much weaker after single-problem learning than after multiple-problem learning, Harlow reasoned that transposition might better be studied in a multiple-problem, learning-set context than in a single-problem context. Research has indeed indicated that size transposition is often greater after nultiple- than after single-problem training (Gonzalez \&z Ross, 1958; Johnson \& Zara, 1960; Sherman \& Strunk, 1964); however, with stimulus size as the only variable, the range of different problems is severely limited. The training which preceded transposition training in the present experiment was a fully developed learning set and probably increased the degree of transposition over what would have occurred after single-problem learning of an ABA vs. ABB discrimination. The flexibility of the learningset technique for transposition testing makes it a promising paradigm for future study.

\section{REFERENCES}

French, G. M. Associative problems. In A. M. Schrier, H. F. Harlow, \& E. Stollnitz (Eds.), Behavior of nonhuman primates (Vol. 1). New York: Academic Press, 1965.

Gonzalez, R. C., \& Ross, S. The basis of solution by preverbal children of the intermediate-size problem. American Journal of Psychology, 1958, 71, 742-746.

HARLow, H. F. Learning set and error factor theory. In S. Koch (Ed.), Psychology: A study of a science (Vol. 2). New York: McGraw-Hill, 1959.

Johnson, R. C., \& Zara, R. C. Relational learning in young children. Journal of Comparative and Physiological Psychology, 1960, 53, 594-597.

KING, J. E. Learning and generalization of a two-dimensional sameness-difference concept by chimpanzees and orangutans. Journal of Comparative and Physiological Psychology, 1973, 84, 140-148.

KING, J. E., \& Fobes, J. L. Hypothesis analysis of samenessdifference learning-set by capuchin monkeys. Learning and Motivation, 1975, 6, 101-113.

Levine, M. Hypothesis behavior. In A. M. Schrier, H. F. Harlow, \& E. Stollnitz (Eds.), Behavior of nonhuman primates (Vol. 1). New York: Academic Press, 1965.

MEYER, D. R., \& HARLOW, H. F. The development of transfer of response to patterning by monkeys. Journal of Comparative and Physiological Psychology, 1949, 42, 454-462.

Miles, R. C. Discrimination-learning sets. In A. M. Schrier, H. F. Harlow, \& E. Stollnitz (Eds.), Behavior of nonhuman primates (Vol. 1). New York: Academic Press, 1965.

REESE, H. W. The penception of stimulus relations. New York: Academic Press, 1968.

Shrrman, M., \& Strunx, J. Transposition as a function of single versus double discrimination training. Journal of Comparative and Physiological Psychology, 1964, 58, 449-450.

SMTtH, H. J., Kinc, J. E., \& NewberRY, P. Facilitation of discrimination learning-set in squirrel monkeys by colored food stimuli. Bulletin of the Psychonomic Society, 1976, 7, 5-8.

SPENCE, $K$. W. The basis of solution by chimpanzees of the intermediate size problem. Joumal of Experimental Psychology, 1942, 31, 257-271.

WARREN, J. M., \& McGonigle, B. Attention theory and discrimination learning. In R. M. Gilbert \& N. S. Sutherland (Eds.), Animal discrimination leaming. New York: Academic Press, 1969.

(Received for publication November 20, 1975; revision accepted March 22, 1976.) 